\title{
Multiple arterial, minimally invasive coronary surgery (MA-MICS)
}

\author{
David R. Hobson, Kevin W. Lobdell, Joseph T. McGinn \\ Sanger Heart \& Vascular Institute, Department of Cardiothoracic and Vascular Surgery, Atrium Health, Charlotte, NC, USA \\ Correspondence to: Kevin W. Lobdell, MD. Sanger Heart \& Vascular Institute, Department of Cardiothoracic and Vascular Surgery, Atrium Health, \\ PO Box 32861, Charlotte, NC 28232, USA. Email: kevin.lobdell@atriumhealth.org.
}

Submitted Feb 01, 2018. Accepted for publication May 27, 2018.

doi: 10.21037/acs.2018.06.03

View this article at: http://dx.doi.org/10.21037/acs.2018.06.03

\section{Introduction}

The morbidity of median sternotomy has led to increasing utilization of minimally invasive techniques in coronary artery bypass surgery (MICS). MICS may also complement the use of multiple arterial grafts for stenotic coronary artery disease (1). Additionally, this technique would appear to circumvent some of the described morbidities of bilateral internal mammary artery harvesting $(2,3)$. Here we present a case of multiple arterial, MICS.

\section{Clinical vignette}

The patient is a 75 -year-old male with severe, multi-vessel coronary artery disease. Stenotic coronary disease risks include type II diabetes mellitus, essential hypertension, hyperlipidemia, and tobacco smoking. Twelve years prior, the patient had percutaneous coronary intervention (PCI) with drug-eluting stents to the left anterior descending (LAD) and right coronary (RCA) arteries. Past medical and surgical history also includes permanent pacemaker, osteoarthritis and gastroesophageal reflux disease.

Recently the patient suffered chest tightness and stress testing demonstrated inferior wall ischemia. 2D echocardiogram demonstrated a left-ventricular ejection fraction of $50 \%$. Coronary angiography revealed $80 \%$ LAD in-stent stenosis, $90 \%$ stenosis of RCA distal to previous stent, and $80 \%$ stenosis of the first obtuse marginal (OM1) and right posterior descending coronary (PDA) arteries.

STS mortality risk was calculated to be $1.27 \%$ and major morbidity risk was $13.6 \%$, hence he was deemed a good surgical candidate. The preoperative plan was to perform minimally invasive coronary bypass via left anterolateral thoracotomy. Specifically, right and left internal mammary artery grafts (RIMA and LIMA) and a sequential, reversed, greater saphenous vein bypass.

\section{Surgical technique}

The patient was positioned supine, upper limbs tucked, and a bolster placed to elevate the patient's left hemithorax. The remainder of the preparation was performed per protocol with both lower limbs and groins prepped and draped into the field. This routine provides access to the femoral vessels should cannulation be needed for cardiopulmonary bypass.

General anesthesia was induced with fentanyl and midazolam. The patient received etomidate and rocuronium prior to endotracheal intubation with an 8.5 endotracheal tube. A bronchial blocker was utilized during the case for single lung ventilation. General anesthesia was maintained with isoflurane gas. Prior to incision, the patient had peripheral venous access placed as well as a right radial arterial line and a right internal jugular pulmonary artery catheter. A transesophageal echocardiography probe was also placed.

A $5 \mathrm{~cm}$ left anterolateral thoracotomy was performed via the fourth intercostal space and additional access was via a subxiphoid incision and a port into the 6th intercostal space. The anterolateral thoracotomy included wide division of the intercostal muscles to facilitate adequate exposure and decrease the retraction force, thereby mitigating the risk of rib fracture. A ThoraTrak ${ }^{\circledR}$ (Medtronic) retractor was used for retraction and a sternal elevator was placed through the subxiphoid access site to assist with skeletonized RIMA harvest, the LIMA was then taken down in a similar fashion from the level of the innominate vein to its bifurcation. Simultaneously, the patient had endoscopic saphenous 
venectomy. The patient then underwent 4 vessel bypass with anastomoses performed in the following chronological order: saphenous vein proximal anastomosis to ascending aorta, RIMA proximal anastomosis end-to-side with LIMA, saphenous vein distal anastomosis to PDA and then in sequence to the posterolateral coronary artery (PL), RIMA distal anastomosis to OM1, and finally LIMA anastomosis to LAD. The aortic anastomosis was performed using a side-biting vascular clamp with careful management of systolic blood pressure.

An $\mathrm{OnQ}^{\mathrm{TM}}$ pump local analgesia infusion catheter was then placed in the fourth intercostal space posteriorly and the thoracotomy was closed in the standard fashion in 3 layers. The total operative time was 5 hours and 8 minutes and the patient was transferred to the intensive care unit (ICU).

\section{Comments}

\section{Clinical outcome}

The patient was liberated from mechanical ventilator support in 4 hours and 41 minutes. The patient was transferred out of the ICU on post-operative day 1 (after removal of chest drains, venous and arterial lines, and urinary catheter) and discharged to home on postoperative day 5. This is similar to described clinical outcomes in this operative population (1). The patient was seen in clinic for scheduled follow up on postoperative day 12 and was noted to be healing well with no residual pleural effusion on chest X-ray.

\section{Advantages}

Multiple arterial grafting has been shown to provide a survival benefit for selected patients over single arterial grafting (2). There is conflicting data on whether the use of multiple arterial grafts in the elderly (age $>70$ ) provides a similar survival benefit to that seen in patients $<70$ years and the ART trial demonstrated elevated rates of sternal wound infection in bilateral mammary arterial harvest (3). Understanding these risks suggests cautious use of bilateral mammary harvest especially in elderly patients. However, these risks are mitigated with the elimination of the sternotomy and thus, multiple arterial bypass may be accomplished without a radial harvest or increased risk of sternal complication. Finally, minimally invasive coronary bypass techniques have been shown to be reproducible and may be implemented safely and effectively (4).

\section{Caveats}

The conceptual foundation of minimally invasive cardiac surgery is the elimination of the morbidity associated with sternotomy. As is true throughout minimally invasive surgery, adequate visualization is key to efficacious use of these techniques. Utilization of an apical stabilizer on the heart allows the surgical field to be brought up immediately underneath the anterolateral thoracotomy. However, posterior vessel targets can still be difficult to access offpump without significant hemodynamic compromise (if they are able to be accessed at all through the $\mathrm{L}$ chest). Judicious use of local anesthesia is also encouraged, as immediate post-operative pain can be significant in these patients. While elimination of the sternotomy alleviates a portion of the morbidity associated with bilateral mammary harvest, continued assessment of ongoing trials $(3,5)$ should provide further insight on which patients most benefit from use of this technique.

\section{Acknowledgements}

None.

\section{Footnote}

Conflicts of Interest: KW Lobdell is an Idependent Quality Consultant for Medtronic; JT McGinn is a Speaker for Medtronic. DR Hobson has no conflicts of interest to declare.

\section{References}

1. Nambiar P, Kumar S, Mittal CM, et al. Minimally invasive coronary artery bypass grafting with bilateral internal thoracic arteries: Will this be the future? J Thorac Cardiovasc Surg 2018;155:190-7.

2. Tranbaugh RF, Schwann TA, Swistel DG, et al. Coronary Artery Bypass Graft Surgery Using the Radial Artery, Right Internal Thoracic Artery, or Saphenous Vein as the Second Conduit. Ann Thorac Surg 2017;104:553-9.

3. Taggart DP, Altman DG, Gray AM, et al. Randomized Trial of Bilateral versus Single Internal-Thoracic-Artery Grafts. N Engl J Med 2016;375:2540-9. 
4. Une D, Lapierre H, Sohmer B, et al. Can minimally invasive coronary artery bypass grafting be initiated and practiced safely?: a learning curve analysis. Innovations (Phila) 2013;8:403-9.

5. Gaudino M, Alexander JH, Bakaeen FG, et al.

Cite this article as: Hobson DR, Lobdell KW, McGinn JT. Multiple arterial, minimally invasive coronary surgery (MAMICS). Ann Cardiothorac Surg 2018;7(4):564-566. doi: 10.21037/acs.2018.06.03
Randomized comparison of the clinical outcome of single versus multiple arterial grafts: the ROMA trialrationale and study protocol. Eur J Cardiothorac Surg 2017;52:1031-40. 